\title{
SUPPLEMENTARY MATERIAL: GAUSSIAN PROCESS MODELING IN APPROXIMATE BAYESIAN COMPUTATION TO ESTIMATE HORIZONTAL GENE TRANSFER IN BACTERIA
}

\author{
By Marko Järvenpä̈̈, Michael U. Gutmann, Aki \\ Vehtari AND PEKKa MartTinen
}

\section{CONTENTS}

1 Additional details and results . . . . . . . . . . . . . . . 1

1.1 Further details on GP models . . . . . . . . . . . . . . 1

1.2 GP model selection for $\mathrm{ABC} \ldots \ldots \ldots 2$

1.3 Posterior estimates compared to the true posterior . . . . . 2

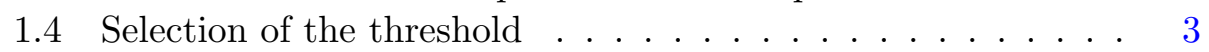

2 Supplementary figures . . . . . . . . . . . . . . . . . . . . . 4

3 Supplementary tables . . . . . . . . . . . . . . . 6

4 Bivariate posterior marginals for the bacterial genomic model . . . 10

\section{Additional details and results.}

1.1. Further details on GP models. We briefly describe the prior densities used for GP hyperparameters $\phi$. Generally speaking, we used rather noninformative priors. Specifically, for the standard GP model, we used the zero mean function i.e. $m(\boldsymbol{\theta})=0$. For the log-transformation, however, we used a small negative mean function because a zero mean function in log-domain would correspond to $m(\boldsymbol{\theta})=\exp (0)=1$ mean function in the original domain. We used t-distribution prior with location 0 , scale half of the range of the parameter space, and degrees of freedom (df) 4 for each of the lengthscale parameters $l_{i}, i=1, \ldots, p$. In general, it can be difficult to know the scale and variation of the discrepancy across the parameter space. Thus we took a pragmatic approach and set a t-distribution prior with location $=0$, scale the standard deviation of the simulated discrepancies (computed using only trimmed values) and $\mathrm{df}=4$ for $\sigma_{f}$. We used an improper uniform prior with support $\mathbb{R}_{+}$for $\sigma^{2}$.

Compared to the standard GP model, the priors for the input-dependent GP model required more careful design to ensure robust computations and we used slightly more informative priors. Zero mean function was used i.e. $m(\boldsymbol{\theta})=0$ (with the exception of log-transformation) as with standard GP. 
As mentioned in the main text, zero mean function was used also for $m_{n}(\boldsymbol{\theta})$. The lengthscale parameters are expected to have rather large values because we expect both the discrepancy and its variance to behave smoothly in all of our test cases. We also assume a priori that the variance of the discrepancy does not vary significantly over the parameter space. Consequently, we used t-distribution priors with the following (hyper)parameters: location: range divided by 3 , scale: range divided by $3, \mathrm{df}=10$ for $l_{f_{i}}$; location: range divided by 2 , scale: range divided by $9, \mathrm{df}=10$ for $l_{g_{i}}$; location: 0 , scale: standard deviation of the simulated discrepancies, $\mathrm{df}=10$ for $\sigma_{f}$; and location $=0$, scale $=1$, $\mathrm{df}=10$ for $\sigma_{g}$.

For the binary GP classification, we set t-distribution prior with location: 0 , scale: the range of parameter space divided by 5 and $\mathrm{df}=4$ for each lengthscale $l_{i}$ and t-distribution prior with location $=0$, scale $=20$ and $\mathrm{df}=4$ for the magnitude $\sigma_{f}$. Also, as discussed in the main text, the MAP estimate for the hyperparameters was used for all these GP models.

1.2. GP model selection for $A B C$. We present the experimental results for the mlpd utility in Figure S1. As discussed in the main text, overall the results look similar to those of the classifier utility, but with some test problems suboptimal GP models are systematically proposed.

Figures S2 and S3 show the GP model selection results when the baseline is the true posterior instead of the $\mathrm{ABC}$ posterior that was used in the main text and in Figure S1. Overall the results are similar but we observe some inconsistencies in the results of both utilities in the case of " $2 \mathrm{D}$ Gaussian 1" and "Lotka-Volterra" test problems. This happens because the log-transformation produces too narrow posterior estimates compared to the corresponding true $\mathrm{ABC}$ posterior and thus poor approximations to it. However, the tendency to produce too narrow posterior estimates compensates the approximation error caused by the nonzero threshold and thus the log-transform produces the best estimates compared to the true posterior.

1.3. Posterior estimates compared to the true posterior. Tables S1 and $\mathrm{S} 2$ contain the results of the experiments in the main text, but the baseline is the true posterior instead of the $\mathrm{ABC}$ posterior. Using the true posterior as a baseline does not change the main conclusions although posterior estimates are worse, as expected, because the nonzero threshold also affects the estimates. However, the additional error caused by the threshold is mostly minor in $1 \mathrm{~d}$ test problems but in $2 \mathrm{~d}$ test problems (especially with LotkaVolterra model in which the discrepancy was a sum of squared errors of individual data points), the posterior estimates are clearly worse. 
1.4. Selection of the threshold. The 0.05th quantile of the simulated discrepancies was chosen for the experiments shown in the main text and for the additional experiments in Sections 1.2 and 1.3. However, we also repeated the experiments with the 0.01th quantile and the corresponding results are shown in Tables S3 and S4. The ABC posterior is used as the baseline here. In general, the results are similar to those with the 0.05th quantile. However, as expected, the classification GP and $\mathrm{ABC}$ rejection sampler methods perform much worse due to the smaller number of discrepancy realisations below the threshold. The computations were also repeated with the 0.1 th quantile and the results were generally similar to those using the 0.05th quantile, except that the error was larger when compared to the true posterior. Consequently, these results are not reported here. 


\section{Supplementary figures.}

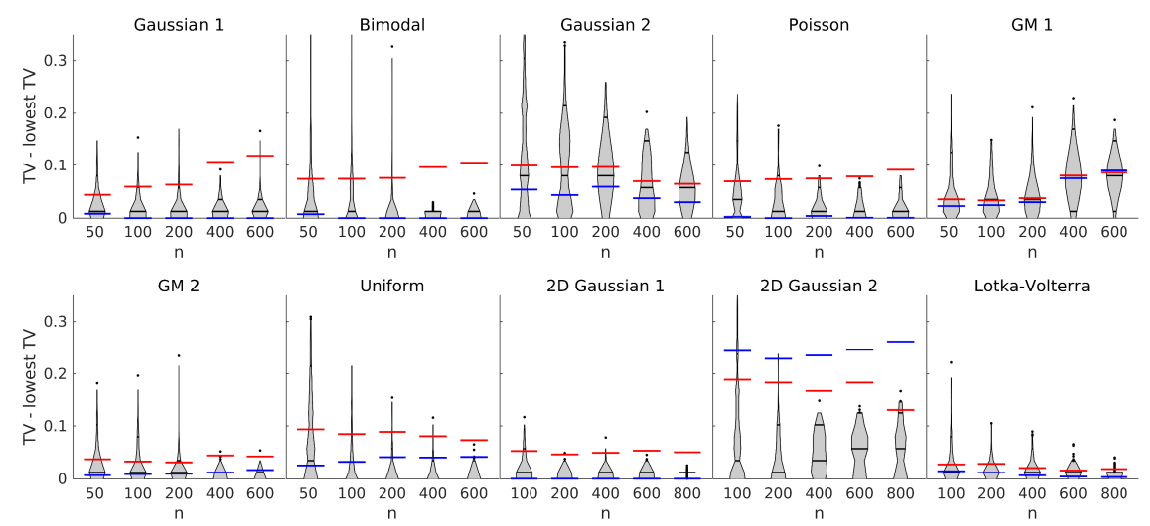

FIG S1. Results of the GP model selection using the mlpd utility. See the caption of the Figure 6 in the main text for details.

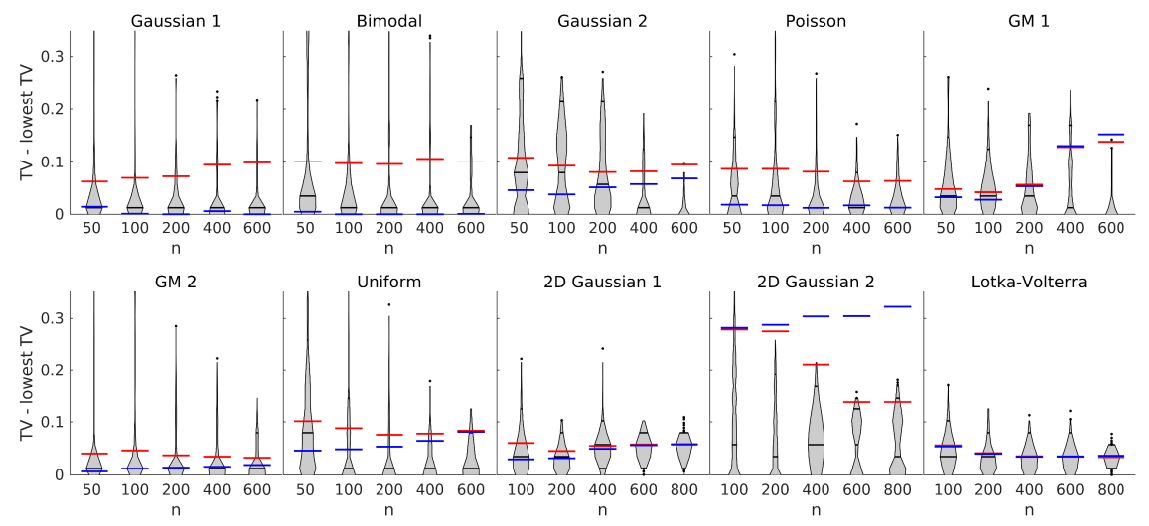

FIG S2. Results of the GP model selection using the classifier utility. The experiments are the same as in Figure $S 1$ expect that comparisons are made to the true posterior. 


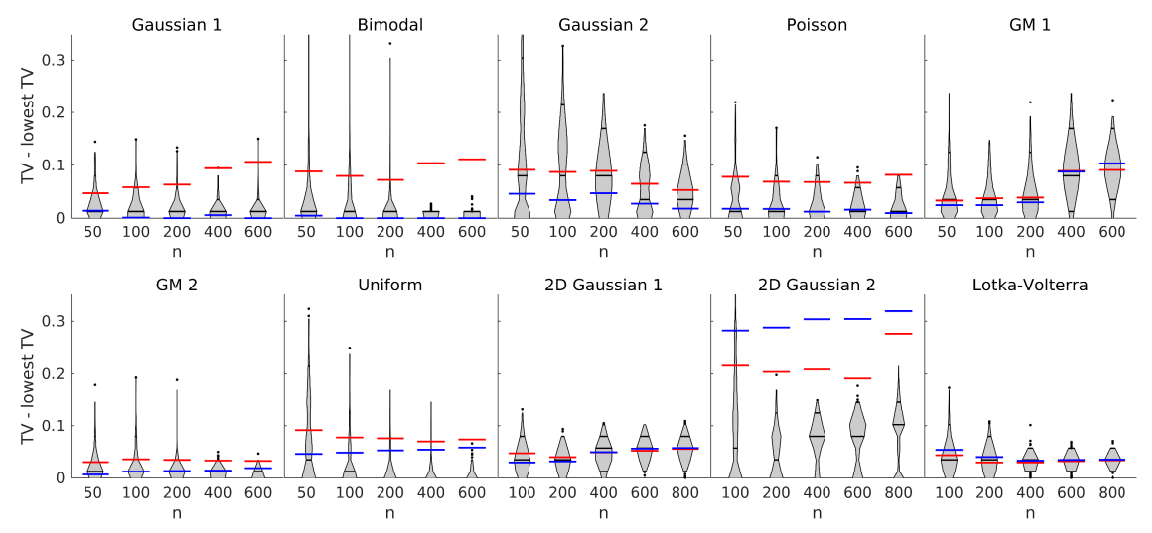

FIG S3. Results of the GP model selection using the mlpd utility. The experiments are the same as in Figure $S 1$ expect that comparisons are made to the true posterior. 


\section{Supplementary tables.}

\begin{tabular}{|c|c|c|c|c|c|c|c|c|c|c|c|c|c|c|c|}
\hline & \multicolumn{3}{|c|}{$\mathrm{n}=50$} & \multicolumn{3}{|c|}{$\mathrm{n}=100$} & \multicolumn{3}{|c|}{$\mathrm{n}=200$} & \multicolumn{3}{|c|}{$\mathrm{n}=400$} & \multicolumn{3}{|c|}{$\mathrm{n}=600$} \\
\hline & se & $\log$ & sqrt & se & $\log$ & sqrt & se & $\log$ & sqrt & se & $\log$ & sqrt & se & $\log$ & sqrt \\
\hline \multicolumn{16}{|l|}{ Gaussian 1: } \\
\hline GP & 0.19 & 0.09 & 0.08 & 0.22 & 0.09 & 0.06 & 0.22 & 0.09 & 0.04 & 0.22 & 0.15 & 0.04 & 0.22 & 0.16 & 0.03 \\
\hline GP in.dep. & 0.19 & 0.13 & 0.09 & 0.18 & 0.13 & 0.06 & 0.19 & 0.11 & 0.05 & 0.20 & 0.12 & 0.04 & 0.23 & 0.12 & 0.04 \\
\hline classifier GP & 0.41 & 0.41 & 0.41 & 0.34 & 0.34 & 0.34 & 0.19 & 0.19 & 0.19 & 0.11 & 0.11 & 0.11 & 0.09 & 0.09 & 0.09 \\
\hline rej. ABC & 0.32 & 0.32 & 0.32 & 0.27 & 0.27 & 0.27 & 0.19 & 0.19 & 0.19 & 0.12 & 0.12 & 0.12 & 0.12 & 0.12 & 0.12 \\
\hline \multicolumn{16}{|l|}{ Bimodal: } \\
\hline GP & 0.22 & 0.48 & 0.16 & 0.21 & 0.26 & 0.12 & 0.22 & 0.17 & 0.10 & 0.22 & 0.15 & 0.07 & 0.22 & 0.16 & 0.07 \\
\hline GP in.dep. & 0.19 & 0.60 & 0.18 & 0.17 & 0.40 & 0.14 & 0.21 & 0.27 & 0.11 & 0.21 & 0.24 & 0.08 & 0.21 & 0.22 & 0.07 \\
\hline classifier GP & 0.41 & 0.41 & 0.41 & 0.35 & 0.35 & 0.35 & 0.25 & 0.25 & 0.25 & 0.18 & 0.18 & 0.18 & 0.14 & 0.14 & 0.14 \\
\hline rej. ABC & 0.46 & 0.46 & 0.46 & 0.40 & 0.40 & 0.40 & 0.26 & 0.26 & 0.26 & 0.21 & 0.21 & 0.21 & 0.17 & 0.17 & 0.17 \\
\hline \multicolumn{16}{|l|}{ Gaussian 2: } \\
\hline GP & 0.31 & 0.37 & 0.26 & 0.31 & 0.24 & 0.25 & 0.32 & 0.21 & 0.24 & 0.32 & 0.22 & 0.23 & 0.31 & 0.23 & 0.22 \\
\hline GP in.dep. & 0.48 & 0.36 & 0.29 & 0.45 & 0.34 & 0.28 & 0.43 & 0.30 & 0.27 & 0.41 & 0.26 & 0.25 & 0.40 & 0.24 & 0.23 \\
\hline classifier GP & 0.35 & 0.35 & 0.35 & 0.34 & 0.34 & 0.34 & 0.25 & 0.25 & 0.25 & 0.18 & 0.18 & 0.18 & 0.15 & 0.15 & 0.15 \\
\hline rej. ABC & 0.28 & 0.28 & 0.28 & 0.29 & 0.29 & 0.29 & 0.21 & 0.21 & 0.21 & 0.17 & 0.17 & 0.17 & 0.15 & 0.15 & 0.15 \\
\hline \multicolumn{16}{|l|}{ GM 1: } \\
\hline GP & 0.35 & 0.32 & 0.33 & 0.33 & 0.30 & 0.32 & 0.32 & 0.30 & 0.32 & 0.32 & 0.23 & 0.31 & 0.31 & 0.20 & 0.31 \\
\hline GP in.dep. & 0.44 & 0.36 & 0.35 & 0.42 & 0.38 & 0.33 & 0.41 & 0.35 & 0.32 & 0.40 & 0.25 & 0.31 & 0.39 & 0.23 & 0.30 \\
\hline classifier GP & 0.36 & 0.36 & 0.36 & 0.37 & 0.37 & 0.37 & 0.29 & 0.29 & 0.29 & 0.19 & 0.19 & 0.19 & 0.15 & 0.15 & 0.15 \\
\hline rej. ABC & 0.37 & 0.37 & 0.37 & 0.34 & 0.34 & 0.34 & 0.25 & 0.25 & 0.25 & 0.20 & 0.20 & 0.20 & 0.16 & 0.16 & 0.16 \\
\hline \multicolumn{16}{|l|}{$\overline{\text { GM } 2:}$} \\
\hline GP & 0.21 & 0.16 & 0.15 & 0.20 & 0.13 & 0.14 & 0.21 & 0.13 & 0.13 & 0.22 & 0.12 & 0.13 & 0.22 & 0.12 & 0.13 \\
\hline GP in.dep. & 0.29 & 0.20 & 0.15 & 0.26 & 0.18 & 0.14 & 0.23 & 0.17 & 0.13 & 0.24 & 0.17 & 0.12 & 0.26 & 0.16 & 0.12 \\
\hline classifier GP & 0.38 & 0.38 & 0.38 & 0.33 & 0.33 & 0.33 & 0.21 & 0.21 & 0.21 & 0.16 & 0.16 & 0.16 & 0.15 & 0.15 & 0.15 \\
\hline rej. ABC & 0.34 & 0.34 & 0.34 & 0.30 & 0.30 & 0.30 & 0.22 & 0.22 & 0.22 & 0.18 & 0.18 & 0.18 & 0.16 & 0.16 & 0.16 \\
\hline \multicolumn{16}{|l|}{ Uniform } \\
\hline GP & 0.28 & 0.19 & 0.17 & 0.29 & 0.20 & 0.16 & 0.29 & 0.19 & 0.17 & 0.29 & 0.20 & 0.17 & 0.29 & 0.20 & 0.17 \\
\hline GP in.dep. & 0.27 & 0.20 & 0.17 & 0.24 & 0.20 & 0.13 & 0.21 & 0.20 & 0.12 & 0.19 & 0.20 & 0.12 & 0.17 & 0.20 & 0.11 \\
\hline classifier GP & 0.44 & 0.44 & 0.44 & 0.35 & 0.35 & 0.35 & 0.23 & 0.23 & 0.23 & 0.14 & 0.14 & 0.14 & 0.10 & 0.10 & 0.10 \\
\hline rej. $\mathrm{ABC}$ & 0.35 & 0.35 & 0.35 & 0.33 & 0.33 & 0.33 & 0.25 & 0.25 & 0.25 & 0.21 & 0.21 & 0.21 & 0.19 & 0.19 & 0.19 \\
\hline \multicolumn{16}{|l|}{ Poisson: } \\
\hline GP & 0.21 & 0.13 & 0.09 & 0.20 & 0.09 & 0.08 & 0.20 & 0.08 & 0.07 & 0.22 & 0.09 & 0.07 & 0.22 & 0.10 & 0.07 \\
\hline GP in.dep. & 0.20 & 0.22 & 0.12 & 0.22 & 0.18 & 0.09 & 0.25 & 0.16 & 0.07 & 0.24 & 0.14 & 0.07 & 0.25 & 0.14 & 0.08 \\
\hline classifier GP & 0.34 & 0.34 & 0.34 & 0.29 & 0.29 & 0.29 & 0.15 & 0.15 & 0.15 & 0.09 & 0.09 & 0.09 & 0.09 & 0.09 & 0.09 \\
\hline rej. $\mathrm{ABC}$ & 0.27 & 0.27 & 0.27 & 0.24 & 0.24 & 0.24 & 0.18 & 0.18 & 0.18 & 0.12 & 0.12 & 0.12 & 0.11 & 0.11 & 0.11 \\
\hline
\end{tabular}

TABLE S1

Results for the $1 D$ toy examples. The numeric values describe the $T V$ distance between the estimated and the corresponding true posterior density. 


\begin{tabular}{|c|c|c|c|c|c|c|c|c|c|c|c|c|c|c|c|}
\hline & \multicolumn{3}{|c|}{$\mathrm{n}=100$} & \multicolumn{3}{|c|}{$\mathrm{n}=200$} & \multicolumn{3}{|c|}{$\mathrm{n}=400$} & \multicolumn{3}{|c|}{$\mathrm{n}=600$} & \multicolumn{3}{|c|}{$\mathrm{n}=800$} \\
\hline & se & $\log$ & sqrt & se & $\log$ & sqrt & se & $\log$ & sqrt & se & $\log$ & sqrt & se & $\log$ & sqrt \\
\hline \multicolumn{16}{|l|}{ 2D Gaussian 1: } \\
\hline GP & 0.35 & 0.19 & 0.19 & 0.34 & 0.15 & 0.18 & 0.33 & 0.11 & 0.16 & 0.33 & 0.10 & 0.16 & 0.33 & 0.10 & 0.15 \\
\hline GP in.dep. & 0.27 & 0.20 & 0.17 & 0.26 & 0.17 & 0.16 & 0.26 & 0.15 & 0.15 & 0.28 & 0.14 & 0.14 & 0.28 & 0.14 & 0.14 \\
\hline classifier GP & 0.69 & 0.69 & 0.69 & 0.70 & 0.70 & 0.70 & 0.29 & 0.29 & 0.29 & 0.21 & 0.21 & 0.21 & 0.19 & 0.19 & 0.19 \\
\hline rej. ABC & 0.43 & 0.43 & 0.43 & 0.35 & 0.35 & 0.35 & 0.30 & 0.30 & 0.30 & 0.27 & 0.27 & 0.27 & 0.25 & 0.25 & 0.25 \\
\hline \multicolumn{16}{|l|}{ 2D Gaussian 2: } \\
\hline GP & 0.62 & 0.44 & 0.56 & 0.62 & 0.39 & 0.54 & 0.61 & 0.35 & 0.52 & 0.61 & 0.34 & 0.52 & 0.61 & 0.33 & 0.52 \\
\hline GP in.dep. & 0.72 & 0.30 & 0.34 & 0.72 & 0.25 & 0.33 & 0.56 & 0.22 & 0.32 & 0.56 & 0.21 & 0.32 & 0.56 & 0.20 & 0.31 \\
\hline classifier GP & 0.70 & 0.70 & 0.70 & 0.71 & 0.71 & 0.71 & 0.44 & 0.44 & 0.44 & 0.28 & 0.28 & 0.28 & 0.24 & 0.24 & 0.24 \\
\hline rej. ABC & 0.45 & 0.45 & 0.45 & 0.40 & 0.40 & 0.40 & 0.34 & 0.34 & 0.34 & 0.32 & 0.32 & 0.32 & 0.31 & 0.31 & 0.31 \\
\hline \multicolumn{16}{|l|}{ Lotka-Volterra: } \\
\hline GP & 0.58 & 0.52 & 0.55 & 0.57 & 0.49 & 0.52 & 0.56 & 0.48 & 0.51 & 0.56 & 0.47 & 0.51 & 0.56 & 0.47 & 0.50 \\
\hline GP in.dep. & 0.65 & 0.50 & 0.53 & 0.56 & 0.48 & 0.51 & 0.55 & 0.47 & 0.50 & 0.55 & 0.47 & 0.50 & 0.55 & 0.47 & 0.50 \\
\hline classifier GP & 0.79 & 0.79 & 0.79 & 0.79 & 0.79 & 0.79 & 0.79 & 0.79 & 0.79 & 0.54 & 0.54 & 0.54 & 0.51 & 0.51 & 0.51 \\
\hline rej. ABC & 0.65 & 0.65 & 0.65 & 0.59 & 0.59 & 0.59 & 0.55 & 0.55 & 0.55 & 0.56 & 0.56 & 0.56 & 0.54 & 0.54 & 0.54 \\
\hline
\end{tabular}

TABLE S2

Results for the $2 D$ toy examples. The numeric values describe the $T V$ distance between the estimated and the corresponding true posterior density. 


\begin{tabular}{|c|c|c|c|c|c|c|c|c|c|c|c|c|c|c|c|}
\hline & \multicolumn{3}{|c|}{$\mathrm{n}=50$} & \multicolumn{3}{|c|}{$\mathrm{n}=100$} & \multicolumn{3}{|c|}{$\mathrm{n}=200$} & \multicolumn{3}{|c|}{$\mathrm{n}=400$} & \multicolumn{3}{|c|}{$\mathrm{n}=600$} \\
\hline & se & $\log$ & sqrt & se & $\log$ & sqrt & se & $\log$ & sqrt & se & $\log$ & sqrt & se & $\log$ & sqrt \\
\hline \multicolumn{16}{|l|}{ Gaussian 1: } \\
\hline GP & 0.18 & 0.20 & 0.08 & 0.21 & 0.23 & 0.06 & 0.21 & 0.24 & 0.05 & 0.21 & 0.32 & 0.04 & 0.21 & 0.32 & 0.03 \\
\hline GP in.dep. & 0.20 & 0.28 & 0.10 & 0.18 & 0.31 & 0.07 & 0.19 & 0.29 & 0.06 & 0.20 & 0.30 & 0.05 & 0.22 & 0.30 & 0.05 \\
\hline classifier GP & 0.51 & 0.51 & 0.51 & 0.56 & 0.56 & 0.56 & 0.56 & 0.56 & 0.56 & 0.52 & 0.52 & 0.52 & 0.39 & 0.39 & 0.39 \\
\hline rej. ABC & 0.60 & 0.60 & 0.60 & 0.60 & 0.60 & 0.60 & 0.37 & 0.37 & 0.37 & 0.26 & 0.26 & 0.26 & 0.20 & 0.20 & 0.20 \\
\hline \multicolumn{16}{|l|}{ Bimodal: } \\
\hline GP & 0.21 & 0.53 & 0.16 & 0.21 & 0.45 & 0.13 & 0.21 & 0.31 & 0.11 & 0.21 & 0.31 & 0.09 & 0.21 & 0.33 & 0.08 \\
\hline GP in.dep. & 0.18 & 0.68 & 0.20 & 0.17 & 0.60 & 0.15 & 0.21 & 0.49 & 0.12 & 0.21 & 0.46 & 0.10 & 0.21 & 0.43 & 0.09 \\
\hline classifier GP & 0.44 & 0.44 & 0.44 & 0.44 & 0.44 & 0.44 & 0.46 & 0.46 & 0.46 & 0.43 & 0.43 & 0.43 & 0.38 & 0.38 & 0.38 \\
\hline rej. ABC & 0.47 & 0.47 & 0.47 & 0.47 & 0.47 & 0.47 & 0.48 & 0.48 & 0.48 & 0.44 & 0.44 & 0.44 & 0.32 & 0.32 & 0.32 \\
\hline \multicolumn{16}{|l|}{ Gaussian 2: } \\
\hline GP & 0.32 & 0.46 & 0.27 & 0.32 & 0.31 & 0.25 & 0.32 & 0.28 & 0.25 & 0.32 & 0.30 & 0.23 & 0.32 & 0.31 & 0.23 \\
\hline GP in.dep. & 0.48 & 0.48 & 0.35 & 0.45 & 0.50 & 0.35 & 0.44 & 0.45 & 0.32 & 0.42 & 0.43 & 0.30 & 0.41 & 0.41 & 0.30 \\
\hline classifier GP & 0.45 & 0.45 & 0.45 & 0.43 & 0.43 & 0.43 & 0.47 & 0.47 & 0.47 & 0.47 & 0.47 & 0.47 & 0.44 & 0.44 & 0.44 \\
\hline rej. ABC & 0.48 & 0.48 & 0.48 & 0.48 & 0.48 & 0.48 & 0.36 & 0.36 & 0.36 & 0.29 & 0.29 & 0.29 & 0.27 & 0.27 & 0.27 \\
\hline \multicolumn{16}{|l|}{ GM 1: } \\
\hline GP & 0.34 & 0.32 & 0.32 & 0.32 & 0.31 & 0.31 & 0.31 & 0.32 & 0.31 & 0.31 & 0.30 & 0.30 & 0.30 & 0.29 & 0.29 \\
\hline GP in.dep. & 0.43 & 0.48 & 0.34 & 0.41 & 0.54 & 0.32 & 0.40 & 0.49 & 0.31 & 0.39 & 0.43 & 0.29 & 0.38 & 0.41 & 0.29 \\
\hline classifier GP & 0.42 & 0.42 & 0.42 & 0.43 & 0.43 & 0.43 & 0.43 & 0.43 & 0.43 & 0.42 & 0.42 & 0.42 & 0.38 & 0.38 & 0.38 \\
\hline rej. ABC & 0.43 & 0.43 & 0.43 & 0.43 & 0.43 & 0.43 & 0.39 & 0.39 & 0.39 & 0.34 & 0.34 & 0.34 & 0.29 & 0.29 & 0.29 \\
\hline \multicolumn{16}{|l|}{ GM 2: } \\
\hline GP & 0.19 & 0.18 & 0.13 & 0.19 & 0.22 & 0.12 & 0.19 & 0.22 & 0.12 & 0.21 & 0.22 & 0.12 & 0.21 & 0.22 & 0.12 \\
\hline GP in.dep. & 0.28 & 0.31 & 0.14 & 0.25 & 0.34 & 0.13 & 0.22 & 0.30 & 0.12 & 0.23 & 0.28 & 0.11 & 0.25 & 0.27 & 0.10 \\
\hline classifier GP & 0.43 & 0.43 & 0.43 & 0.46 & 0.46 & 0.46 & 0.47 & 0.47 & 0.47 & 0.47 & 0.47 & 0.47 & 0.33 & 0.33 & 0.33 \\
\hline rej. ABC & 0.49 & 0.49 & 0.49 & 0.49 & 0.49 & 0.49 & 0.36 & 0.36 & 0.36 & 0.28 & 0.28 & 0.28 & 0.25 & 0.25 & 0.25 \\
\hline \multicolumn{16}{|l|}{ Uniform } \\
\hline GP & 0.27 & 0.35 & 0.16 & 0.28 & 0.38 & 0.16 & 0.28 & 0.37 & 0.17 & 0.28 & 0.38 & 0.17 & 0.28 & 0.38 & 0.17 \\
\hline GP in.dep. & 0.27 & 0.31 & 0.17 & 0.23 & 0.33 & 0.13 & 0.21 & 0.35 & 0.13 & 0.19 & 0.39 & 0.12 & 0.18 & 0.38 & 0.12 \\
\hline classifier GP & 0.45 & 0.45 & 0.45 & 0.49 & 0.49 & 0.49 & 0.51 & 0.51 & 0.51 & 0.51 & 0.51 & 0.51 & 0.48 & 0.48 & 0.48 \\
\hline rej. ABC & 0.51 & 0.51 & 0.51 & 0.51 & 0.51 & 0.51 & 0.42 & 0.42 & 0.42 & 0.33 & 0.33 & 0.33 & 0.30 & 0.30 & 0.30 \\
\hline \multicolumn{16}{|l|}{ Poisson: } \\
\hline GP & 0.20 & 0.21 & 0.10 & 0.19 & 0.25 & 0.08 & 0.20 & 0.25 & 0.07 & 0.21 & 0.29 & 0.06 & 0.21 & 0.31 & 0.07 \\
\hline GP in.dep. & 0.20 & 0.39 & 0.14 & 0.21 & 0.40 & 0.12 & 0.26 & 0.38 & 0.09 & 0.25 & 0.37 & 0.10 & 0.26 & 0.38 & 0.10 \\
\hline classifier GP & 0.45 & 0.45 & 0.45 & 0.50 & 0.50 & 0.50 & 0.38 & 0.38 & 0.38 & 0.28 & 0.28 & 0.28 & 0.18 & 0.18 & 0.18 \\
\hline rej. ABC & 0.57 & 0.57 & 0.57 & 0.43 & 0.43 & 0.43 & 0.26 & 0.26 & 0.26 & 0.19 & 0.19 & 0.19 & 0.16 & 0.16 & 0.16 \\
\hline
\end{tabular}

TABLE S3

Results for the $1 D$ toy examples when the threshold is 0.01th quantile. The numeric values describe the $T V$ distance between the estimated and the corresponding $A B C$ posterior density. 


\begin{tabular}{|c|c|c|c|c|c|c|c|c|c|c|c|c|c|c|c|}
\hline & \multicolumn{3}{|c|}{$\mathrm{n}=100$} & \multicolumn{3}{|c|}{$\mathrm{n}=200$} & \multicolumn{3}{|c|}{$\mathrm{n}=400$} & \multicolumn{3}{|c|}{$\mathrm{n}=600$} & \multicolumn{3}{|c|}{$\mathrm{n}=800$} \\
\hline & se & $\log$ & sqrt & se & $\log$ & sqrt & se & $\log$ & sqrt & se & $\log$ & sqrt & se & $\log$ & sqrt \\
\hline \multicolumn{16}{|l|}{ 2D Gaussian 1: } \\
\hline GP & 0.29 & 0.29 & 0.12 & 0.28 & 0.28 & 0.09 & 0.27 & 0.28 & 0.07 & 0.27 & 0.28 & 0.07 & 0.27 & 0.29 & 0.06 \\
\hline GP in.dep. & 0.24 & 0.41 & 0.16 & 0.24 & 0.33 & 0.11 & 0.23 & 0.28 & 0.09 & 0.25 & 0.27 & 0.08 & 0.25 & 0.27 & 0.07 \\
\hline classifier GP & 0.65 & 0.65 & 0.65 & 0.67 & 0.67 & 0.67 & 0.67 & 0.67 & 0.67 & 0.67 & 0.67 & 0.67 & 0.67 & 0.67 & 0.67 \\
\hline rej. ABC & 0.68 & 0.68 & 0.68 & 0.68 & 0.68 & 0.68 & 0.37 & 0.37 & 0.37 & 0.34 & 0.34 & 0.34 & 0.31 & 0.31 & 0.31 \\
\hline \multicolumn{16}{|l|}{ 2D Gaussian 2: } \\
\hline GP & 0.59 & 0.38 & 0.52 & 0.59 & 0.28 & 0.49 & 0.58 & 0.25 & 0.47 & 0.57 & 0.25 & 0.46 & 0.57 & 0.25 & 0.46 \\
\hline GP in.dep. & 0.69 & 0.50 & 0.41 & 0.67 & 0.39 & 0.37 & 0.59 & 0.35 & 0.36 & 0.59 & 0.31 & 0.37 & 0.59 & 0.29 & 0.35 \\
\hline classifier GP & 0.68 & 0.68 & 0.68 & 0.68 & 0.68 & 0.68 & 0.69 & 0.69 & 0.69 & 0.69 & 0.69 & 0.69 & 0.68 & 0.68 & 0.68 \\
\hline rej. ABC & 0.70 & 0.70 & 0.70 & 0.70 & 0.70 & 0.70 & 0.41 & 0.41 & 0.41 & 0.36 & 0.36 & 0.36 & 0.32 & 0.32 & 0.32 \\
\hline \multicolumn{16}{|l|}{ Lotka-Volterra: } \\
\hline GP & 0.28 & 0.24 & 0.25 & 0.24 & 0.20 & 0.20 & 0.21 & 0.18 & 0.18 & 0.20 & 0.17 & 0.16 & 0.19 & 0.16 & 0.14 \\
\hline GP in.de & 0.53 & 0.31 & 0.30 & 0.29 & 0.27 & 0.24 & 0.25 & 0.23 & 0.20 & 0.24 & 0.22 & 0.18 & 0.22 & 0.20 & 0.16 \\
\hline classifier GP & 0.59 & 0.59 & 0.59 & 0.60 & 0.60 & 0.60 & 0.59 & 0.59 & 0.59 & 0.59 & 0.59 & 0.59 & 0.59 & 0.59 & 0.59 \\
\hline rej. ABC & 0.60 & 0.60 & 0.60 & 0.60 & 0.60 & 0.60 & 0.35 & 0.35 & 0.35 & 0.31 & 0.31 & 0.31 & 0.29 & 0.29 & 0.29 \\
\hline
\end{tabular}

TABLE S4

Results for the 2D toy examples when the threshold is the 0.01th quantile. The numeric values describe the $T V$ distance between the estimated and the corresponding $A B C$ posterior density. 


\section{Bivariate posterior marginals for the bacterial genomic model.}

The bivariate marginal posteriors for the bacterial genomic model described in Section 3.3 of the main text are shown in Figure S4.
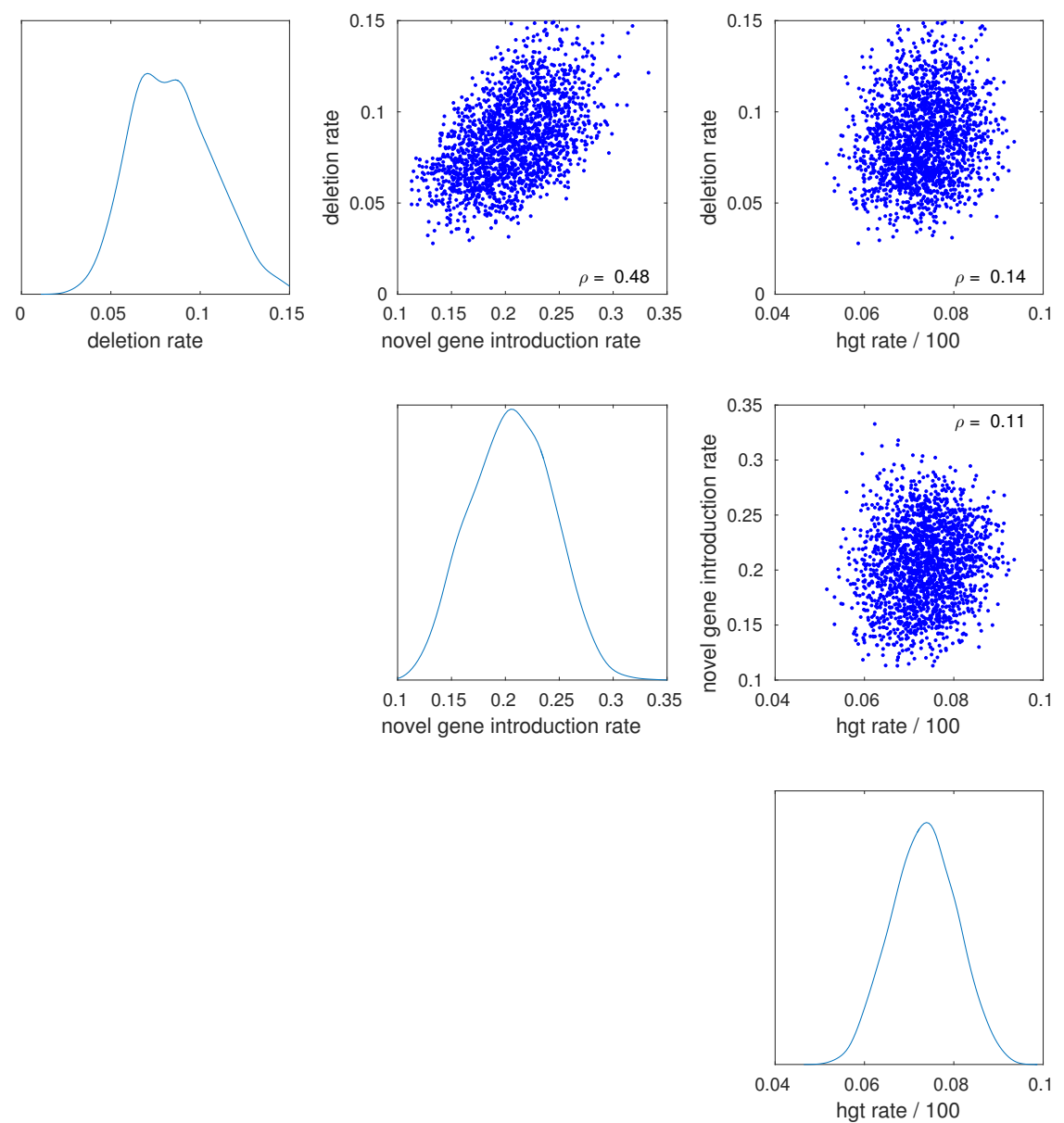

FIG S4. Estimated posterior distribution for the bacterial genomic model. The value of $\rho$ is the (Pearson) correlation coefficient between the corresponding parameters of the model. 